\title{
Lesedocks für Kinder im Spital
}

\author{
Kreativität und Kommunikation fördern die Gesundheit.
}

\begin{abstract}
Mehr als 22.000 junge Patienten wurden 2007 in den Kinder- und Jugendabteilungen sowie Kinder- und Jugendpsychiatrien der NÖ Landeskliniken-Holding behandelt. Ihre hauptsächliche Freizeitbeschäftigung: Fernsehen. Als abwechslungsreiche Alternative bieten Zeit Punkt Lesen - Leseland Niederösterreich und die NÖ Landeskliniken-Holding nun LESEDOCKS.
\end{abstract}

Diese Lesestationen mit Sprachund Zeichenspielen sowie Büchern sollen Unterhaltung in den Tagesablauf im Spital bringen. Kreativität, Lachen und gemeinsame Beschäftigung baut Ängste ab, steigert das Wohlbefinden und fördert letztlich den Heilungsprozess. Alle elf

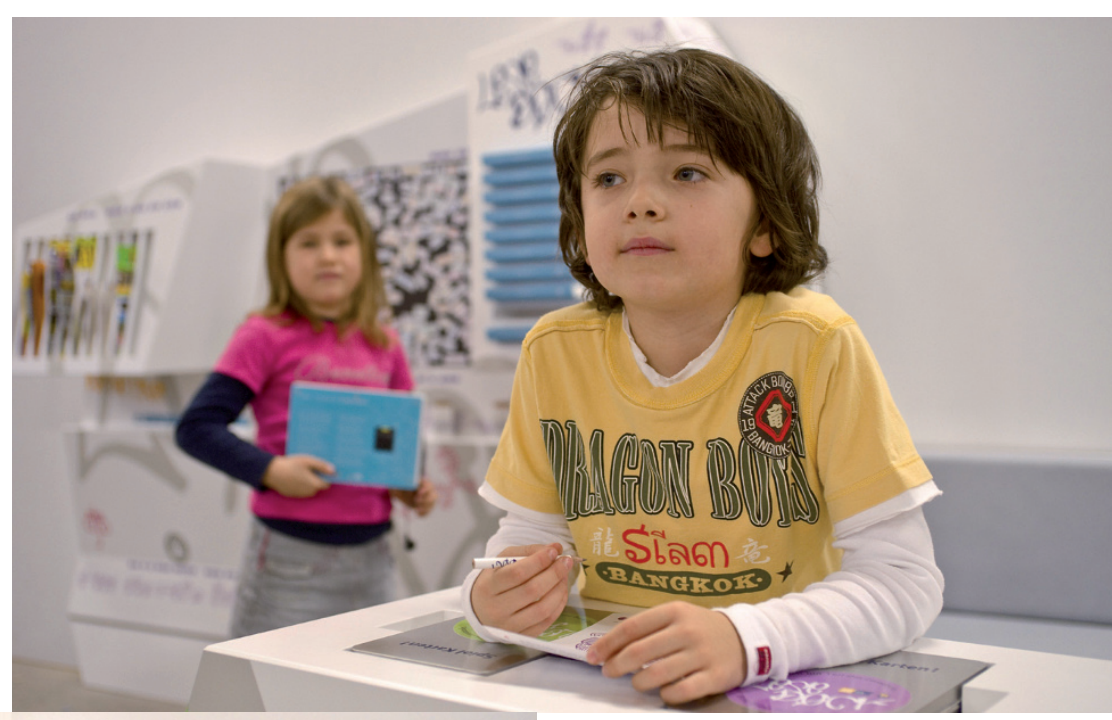

Magnetbuchstaben Zeichen setzen, sich in Morse-, Graffiti- und Fingeralphabet üben oder Worträtsel lösen. Für Leseratten hat der Verlag Carl Ueberreuter Bücher und Magazine zur Verfügung gestellt. Auch der Märchenerzähler Folke Tegetthoff konnte für das Projekt gewonnen werden. Er stellte Geschichten aus aller Welt zu speziellen MP3-,ZZauberbüchern“ zusammen. Um jungen Patienten in der Ambulanz und in den Betten die Zeit im Spital zu verkürzen, wurden die Lesedocks um drei mobile Wägen, die Ausflugsboote, ergänzt. Sie bringen die Angebote der Lesedocks direkt zu den Kindern und Jugendlichen.

„Mit dem Lesedock entsteht ein neuer Ort in der Abteilung, der neue Möglichkeiten eröffnet, so Dr. Hans Salzer, Leiter der Kinder- und Jugendabteilung am Landesklinikum Donauregion Tulln. Das wird sich auch im Stationsalltag widerspiegeln." Nicht nur Schwellenängste, auch Sprachbarrieren lassen sich damit überwinden. Türkischsprachige Kinder und Eltern können mit Hilfe eigens angefertigter "Spitalssprachführer" leichter mit den Krankenhausmitarbeitern in Kontakt treten. Und deutschsprachige Kinder können bei ihren türkischsprachigen Bettnachbarn „Nachhilfe“ nehmen. Informationen: www.zeitpunktlesen.at 